\title{
Enteral Feeding Reduces Endothelial Nitric Oxide Synthase in the Caudal Intestinal Microvasculature of Preterm Piglets
}

\author{
ELS R. VAN HAVER, MARIJKE OSTE, THOMAS THYMANN, STANISLAS U. SYS, WOUTER H. LAMERS, \\ ANDRE L.M. WEYNS, PER T. SANGILD, AND CHRISTA J. VAN GINNEKEN
}

\begin{abstract}
Department of Veterinary Medicine [E.R.H, M.O., A.L.M.W., C.J.G.], University of Antwerp, 2610 Wilrijk, Belgium; Department of Human Nutrition [T.T., P.T.S.], University of Copenhagen, Frederiksberg DK-1958, Denmark; Department of Pharmacology, Toxicology, Biochemistry and Organ Physiology [S.U.S.], University of Ghent, 9820 Merelbeke, Belgium; Department of Anatomy and Embryology
\end{abstract} [W.H.L.], University of Maastricht, 6200 MD Maastricht, The Netherlands

\begin{abstract}
The initiation of enteral feeding represents a challenge to the neonatal intestinal microcirculation, especially in preterms where it predisposes to necrotizing enterocolitis (NEC). We hypothesized that a structural microvascular deficiency may occur when enteral feeding is initiated in preterm piglets susceptible to NEC. Stereologic volume densities of a pan-endothelial marker (vWF), and the main vasodilator endothelial nitric oxide synthase (eNOS), were determined along the small intestine of 1) unfed preterm piglets, 2) piglets receiving total parenteral nutrition (TPN) for 2-3 d, and 3) piglets fed $2 \mathrm{~d}$ sow's colostrum (TPN+SOW) or milk formula (TPN+FOR) following TPN. In the mucosa, vWF-density decreased in a cranio-caudal direction. A corresponding mucosal eNOS gradient appeared only after initiating enteral feeding. In TPN+SOW, eNOS induction may lag behind the mucosal growth of the caudal region. In TPN+FOR, formula-related factors (i.e. bacteria, cytokines) may suppress mucosal eNOS, indicated by increased stresssensitive nuclear HIF1 $\alpha$ staining. The low mucosal endothelial eNOS density was related to the presence of NEC lesions, maybe via increased hypoxia-sensitivity, especially in the caudal region as indicated by nuclear HIF1 $\alpha$-staining. Our results suggest an insufficient structural adaptation of the microvasculature to enteral feeding, especially of mucosal eNOS, which may lead to NEC. (Pediatr Res 63: 137-142, 2008)
\end{abstract}

$\mathrm{T}$ he birth process converts the relatively dormant fetal intestine into an organ of intense metabolic activity and associated abundant perfusion, as it becomes the sole site for nutrient absorption. This profound transition is particularly problematic at premature birth, where it may result in NEC, a severe inflammatory bowel disease associated with the initiation of enteral feeding, especially formula (1-3). Therefore, a period of total parenteral nutrition (TPN) in preterm infants is often required before enteral feeding is tolerated (4). The etiology of NEC is unknown, but a dysfunctional intestinal microcirculation could contribute to the etiopathogenesis $(5,6)$.

The newborn intestinal circulation shows some unique features, up to now intensely studied from a functional point of

Received July 2, 2007; accepted September 19, 2007.

Correspondence: Chris Van Ginneken, D.V.M., Ph.D., Laboratory of Veterinary Anatomy and Embryology, Universiteitsplein 1, 2610 Wilrijk, Belgium; e-mail: Chris.vanginneken@ua.ac.be

This research was supported by a Special Research Fund of the University of Antwerp and by a Research Grant of the Fund for Scientific Research-Flanders Belgium (1.5.096.04N). view $(5,7)$. Nitric oxide (NO), a gaseous free radical produced mainly by endothelial nitric oxide synthase (eNOS), is the most important vasodilator during the perinatal period in sheep (8) and pig (9). Although eNOS is constitutively expressed in the endothelium, both eNOS mRNA and protein expression can be modulated (10), including by nutrient intake (7). Furthermore, intestinal blood flow shows a cranial-tocaudal decrease along the small intestine in neonatal and adult dogs (11), pigs $(12,13)$, and humans (14). It is, however, unknown whether this functional gradient in the small intestine is associated with a similar gradient in microvessel distribution and/or eNOS protein expression. Histologic analyses of the regional morphometrics of the intestinal endothelium and eNOS expression during the first days of life are therefore interesting. Further, we do not know if there is a relation to tissue hypoxia during this critical time. Nuclear hypoxia inducible factor-1 immunoreactivity by immunohistochemistry (HIF1 $\alpha$-IR) can be used as an indicator for such cellular stress, although it is potentially induced by bacterial lipopolysaccharide (LPS) and cytokines as well $(15,16)$. We hypothesized that the response of these parameters to enteral feeding in preterm neonates would be consistent with an increased NEC sensitivity. Therefore, our aim was dual: first, to characterize structural changes in the microvasculature and its eNOS expression along the small intestine in premature pigs fed similarly as premature infants. Second, to use these data to indicate hypoxia sensitivity due to retarded endothelial development in a premature piglet model of NEC.

\section{MATERIALS AND METHODS}

Animals. Surgery, delivery, assignment to feeding patterns, and incubation of the premature piglets were carried out according to methods described earlier (17). In short, 34 piglets from 6 sows (Large White $\times$ Danish Landrace) were delivered by cesarean section at 93-94\% of gestation. After delivery, the piglets were placed in infant incubators with regulated temperature, moisture, and oxygen supply. Rectal temperature and arterial $\mathrm{Hb}$ saturation were monitored. The experiments were approved by the National 
Committee on Animal Experimentation, Denmark, and the Ethical Committee on Animal Experimentation, University of Antwerp, Belgium.

The piglets were randomly divided into four groups. One group of newborn piglets was killed within $12 \mathrm{~h}$ of birth and received no nutrition (NB, $n=5$ ). All other pigs were given TPN (Nutriflex Lipid plus, Braun, Melsungen, Germany). The composition of Nutriflex Lipid plus was modified as described in previous studies (17) to provide sufficient energy and protein for a slightly positive energy balance. The TPN solution was infused continuously at $4-8 \mathrm{~mL} / \mathrm{kg} / \mathrm{h}$ for $3 \mathrm{~d}$ via a catheter inserted into the transected umbilical artery using automatic infusion pumps (Infusomat Secura, Braun). After 2-3 d of TPN, a group of piglets was killed (TPN, $n=10$ ), whereas the rest started with total enteral nutrition with either sow's colostrum (TPN + SOW, $n=9$ ) or infant milk formula (TPN+FOR, $n=10)$ collected and prepared as described previously $(3,17)$. Both diets were fed at 15 $\mathrm{mL} / \mathrm{kg} / 3 \mathrm{~h}$ for $32-42 \mathrm{~h}$ after the TPN period.

Tissue collection. All piglets were anesthetized (Zoletil 50, Boehringer Ingelheim, Copenhagen, Denmark) and subsequently killed in a fasting state with an intracardiac injection of sodium pentobarbitone (Nembutal, Abbott Laboratories, Chicago, IL). The small intestine was rapidly excised and divided into three segments of equal length, designated cranial, middle, and caudal region. Tissue samples of 2-3 cm were removed from the middle of each region, rinsed, and fixed for $2 \mathrm{~h}$ in $4 \%$ freshly prepared formaldehyde (0.1 M, pH 7.4). The fixative was subsequently washed out with $0.01 \mathrm{M}$ PBS for $24 \mathrm{~h}$ and tissues were stored in PBS containing $0.01 \% \mathrm{NaN}_{3}$ at $4{ }^{\circ} \mathrm{C}$.

Paraffin sections. From each tissue sample, one biopsy (3-5 $\mathrm{mm}$ diameter) was taken, embedded in paraffin, and sliced in $5 \mu \mathrm{m}$ vertical paraffin sections. The first section was collected randomly and subsequent sections were collected a systematic distance of 4 sections apart, giving 10 sections per small intestinal region per pig used for morphometry.

Hematoxylin-eosin sections. The HE sections were used for estimating villus width, crypt depth, and villus height. These parameters were used in a mathematical formula to estimate absolute mucosal/villus growth. Considering the fingerlike villus shape in neonatal piglets (18), villi may be regarded as approaching a cylindrical shape, thus villus volume was estimated by $\pi *$ (width $/ 2)^{2} *$ (villus height + crypt depth).

Histopathologic scoring of the intestinal NEC lesions was performed on HE sections according to absence (score 0 ) or presence of lesions (score 1) (Fig. 1). Lesions were characterized by clear tissue damage, either necrosis of the villi, severe congestion with hemorrhage and/or separation of the tela submucosa.
Immunohistochemistry. Briefly, the basic immunohistochemical protocols were as follows (Table 1). After deparaffinization, antigen retrieval was performed. Nonspecific staining was blocked successively by incubation in $3 \% \mathrm{H}_{2} \mathrm{O}_{2}$ and $10 \%$ normal rabbit serum. Subsequently, primary antibodies and commercial enzyme-conjugated secondary antibodies were used, after which the immune complexes were visualized.All dilutions were made in $0.01 \%$ Tris-buffered saline ( $\mathrm{pH}$ 7.4) with $0.3 \%$ Triton- $\times 100$, and washings in Tris-buffered saline were performed between different steps. Positive control slides for HIF1 $\alpha$ consisted of 45-min ischemic intestinal loops of newborn piglets.

Quantitative morphologic analyses. An Olympus BX50 microscope, equipped with a DP70 camera connected to a computer system running the software program Cast-grid (Olympus), was used for the quantitative analyses. The optimal density of the stereological grid, the number of sections, and the number of sample fields were estimated as described previously (19). The volume density (Vv) of eNOS and von Willebrandt factor (vWF) in the different layers of the intestinal wall were estimated by using a grid overlay on a series of blinded, immunohistochemically stained sections and subsequently counting the number of grid points $(\mathrm{Q})$ within the immunoreactive area $[\mathrm{Q}(\mathrm{Y})]$ and the number of grid points within the intestinal wall $[\mathrm{Q}(\mathrm{ref})]$. The following equation yields the volume density expressed as percentage: $\mathrm{Vv}(\mathrm{Y} ; \mathrm{ref})=[\mathrm{Q}(\mathrm{Y}) / \mathrm{Q}(\mathrm{ref})]^{* 100}($ Table 2$)$.

In addition, a new variable was created to express the ratio of eNOS-IR to vWF-IR in the mucosal endothelium (eNOS/vWF). As vWF is present in all endothelial cells, this variable determines whether observed differences in eNOS Vvmuc are due to alterations in endothelial cell distribution (vWF) in the mucosa or to eNOS expression within the endothelium. Finally, nuclear HIF $1 \alpha$ was microscopically evaluated as being present or absent.

Statistical evaluation (using SPSS). Three samples were excluded from analyses as their villi were too severely damaged. All stereological data were arcsine-transformed to obtain nearly normal underlying distributions. They were analyzed by repeated measures ANOVA to detect significant effects of intestinal region or feeding regimen on vWF-IR, eNOS-IR, villus width, and mucosal growth. Post hoc comparisons were performed using an LSD test for diet effects and a Scheffé test for effects of intestinal region. Standard Pearson-correlation testing was performed to evaluate associations between villus width and vWF-IR. Independent sample $t$ tests were calculated to determine whether vWF Vvmuc or eNOS Vvmuc depended on lesion score or hypoxia. A relation between nuclear HIF1 $\alpha$ staining and feeding regimen or region was evaluated by means of a $\chi^{2}$ test. Results are expressed as means \pm $\operatorname{SEM} p<0.05$ were considered significant.
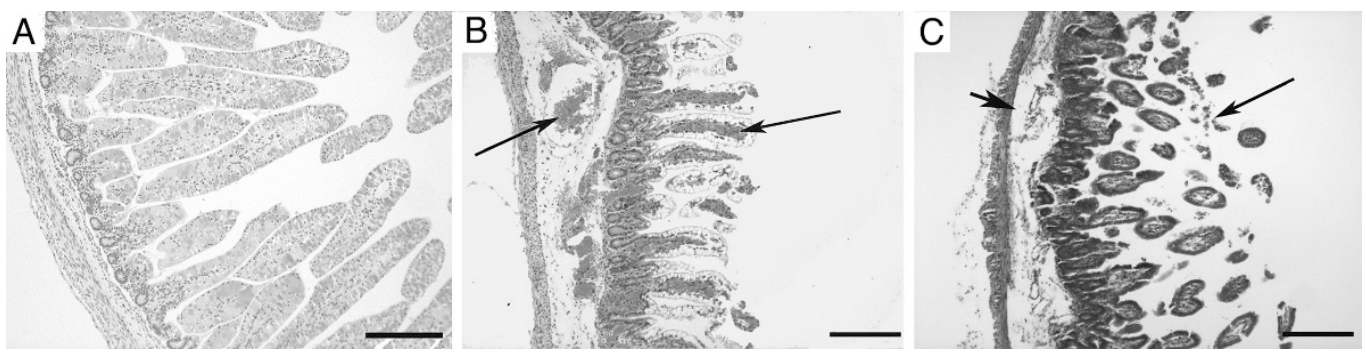

Figure 1. Scoring of NEC-like lesions. HE-stained sections of small intestine from preterm pigs, illustrating score 0 -tissue $(A)$, and score $1-$ tissue $(B$, $C$ ). Lesions were characterized by necrosis of the villi, severe congestion with hemorrhage (thin arrows), and/or separation of the tela submucosa (thick arrow). Scale bar $=200 \mu \mathrm{m}$.

Table 1. Immunohistochemical protocols

\begin{tabular}{|c|c|c|c|c|}
\hline Target & Antigen retrieval & Primary antibody & Secondary antibody & Visualization \\
\hline $\begin{array}{l}\text { Endothelial } \\
\text { cells }\end{array}$ & $\begin{array}{l}\text { Enzymatic digestion } \\
\quad(\text { trypsin } 0.05 \%)\end{array}$ & $\begin{array}{l}\text { Anti-vWF (Dako, Glostrup, } \\
\text { Denmark) } 1: 50037^{\circ} \mathrm{C}, 90 \\
\text { min }\end{array}$ & Anti-rabbit envision (Dako) & AEC (Dako) \\
\hline $\begin{array}{l}\text { Endothelial } \\
\text { eNOS }\end{array}$ & $\begin{array}{l}\text { TRIS pH 10, pressure } \\
\text { cooker }\end{array}$ & $\begin{array}{l}\text { Anti-eNOS (BD Transduction } \\
\text { Laboratories, Franklin } \\
\text { Lakes, NJ) 1:500 } 37^{\circ} \mathrm{C}, 90 \\
\text { min }\end{array}$ & $\begin{array}{l}\text { PowerVision (ImmunoLogic, } \\
\text { Duiven, the Netherlands) }\end{array}$ & $\begin{array}{l}\text { NBT/PCIB (Roche, } \\
\text { Basel, Switzerland) }\end{array}$ \\
\hline $\begin{array}{l}\text { Hypoxic } \\
\text { cells }\end{array}$ & $\begin{array}{l}\text { TRIS-EDTA pH 9, } \\
\text { microwave }\end{array}$ & $\begin{array}{l}\text { Anti-HIF1 } \alpha \text { (Novus } \\
\text { Biologicals, Littleton, CO) } \\
1: 1004^{\circ} \mathrm{C}, 20 \mathrm{~h}\end{array}$ & Anti-mouse envision (Dako) & AEC (Dako) \\
\hline
\end{tabular}

Immunohistochemistry for $\mathrm{vWF}$, eNOS, and HIF1 $\alpha$ consisted of subsequent application of antigen retrieval, and primary and secondary antibody incubation. Secondary antibodies were applied according to the manufacturer's recommendations. AEC, aminoethylcarbazole; NBT/PCIB, nitro blue tetrazolium/5-bromo4chloro3-indoyl phosphate. 
Table 2. Stereologic volume densities

\begin{tabular}{|c|c|c|c|}
\hline & Vv & $\begin{array}{c}\text { Immunoreactivity } \\
\text { (numerator) }\end{array}$ & $\begin{array}{l}\text { Intestinal wall } \\
\text { (denominator) }\end{array}$ \\
\hline \multirow[t]{3}{*}{ eNOS } & Vvmuc & $\begin{array}{l}\text { Mucosal eNOS } \\
\text { volume }\end{array}$ & Mucosal volume \\
\hline & Vvsubmuc & $\begin{array}{l}\text { Submucosal eNOS } \\
\text { volume }\end{array}$ & $\begin{array}{c}\text { Submucosal } \\
\text { volume }\end{array}$ \\
\hline & Vvmusc & $\begin{array}{l}\text { Muscular eNOS } \\
\text { volume }\end{array}$ & Muscular volume \\
\hline \multirow[t]{3}{*}{ vWF } & Vvmuc & $\begin{array}{l}\text { Mucosal vWF } \\
\text { volume }\end{array}$ & Mucosal volume \\
\hline & Vvsubmuc & $\begin{array}{l}\text { Submucosal vWF } \\
\text { volume }\end{array}$ & $\begin{array}{l}\text { Submucosal } \\
\text { volume }\end{array}$ \\
\hline & Vvmusc & $\begin{array}{l}\text { Muscular vWF } \\
\text { volume }\end{array}$ & Muscular volume \\
\hline eNOS/vWF & Vvmuc & $\begin{array}{l}\text { Mucosal eNOS } \\
\text { volume }\end{array}$ & $\begin{array}{l}\text { Mucosal vWF } \\
\text { volume }\end{array}$ \\
\hline
\end{tabular}

Estimated volume densities of eNOS, vWF, and eNOS/vWF in various layers of the intestinal wall. Volume density represents a ratio of volume of immunoreactivity versus a reference volume, i.e. the intestinal wall.

\section{RESULTS}

Microscopic evaluation. VWF-IR (a pan endothelial marker) and eNOS-IR were exclusively seen in the cytoplasm of endothelial cells delineating the blood vessels in all layers of the intestinal wall (Fig. 2). In the muscle layer, most blood vessels were observed running between the longitudinal and circular muscle layer, sometimes extending between the muscle bundles. The largest vessels were located in the submucosal layer, where the venules showed variable eNOS staining. The mucosal vessels were eNOS- and vWF-IR from the base of the villus to the top and they were seen as two to three parallel strings running along the villus axis (Fig. 2). These vessels were often localized close to the mucosal epithelium, in accordance with the classical "fountain pattern" described in pigs (20). In some samples, eNOS-IR faded toward the villus tips, independent of feeding regimen or region (percentage of samples showing fading gradient: cranial region $=20.8 \%$,

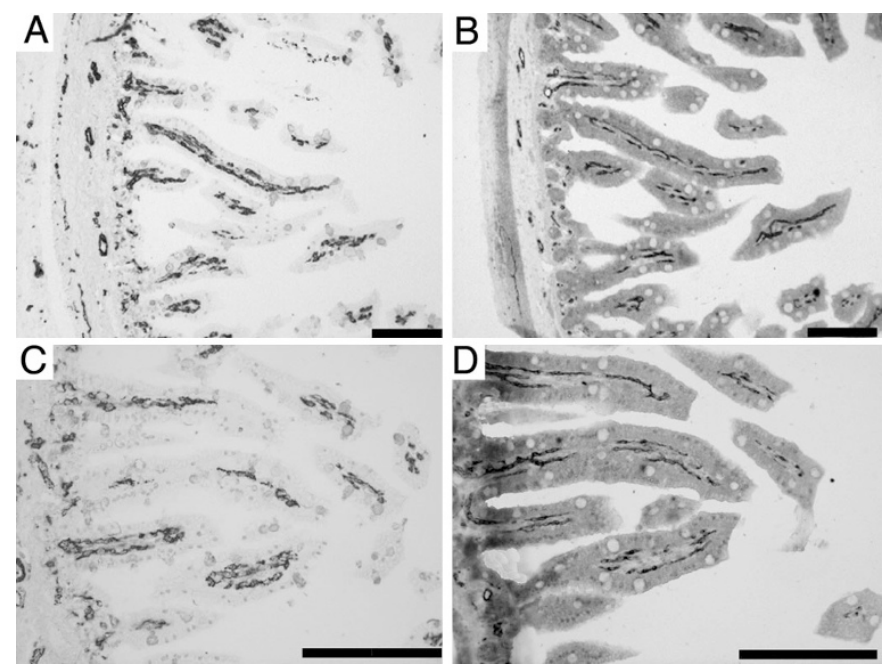

Figure 2. Comparable eNOS and vWF immunohistochemistry. Immunoreactivity for eNOS $(A, C)$ and $\mathrm{vWF}(B, D)$ in newborn piglets. Mucosal blood vessels are visible as strings running parallel to the villus axis. A larger volume of vWF-IR compared with eNOS-IR can be observed in the same villus volume. Scale bar $=250 \mu \mathrm{m}$.

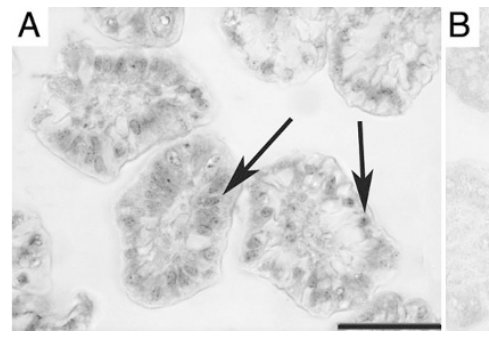

Figure 3. Immunoreactivity for $\operatorname{HIF} 1 \alpha$. Staining of $\operatorname{HIF} 1 \alpha$ in villi of a pig receiving formula- $(A)$ or colostrum-feeding $(B)$. Formula-fed piglets often showed nuclear immunoreactivity (arrows) indicative for cellular stress, such as hypoxia. Scale bar $=50 \mu \mathrm{m}$.

middle region $=31.0 \%$, caudal region $=21.4 \%, \mathrm{NB}=15.4 \%$, $\mathrm{TPN}=50.0 \%, \mathrm{TPN}+\mathrm{SOW}=26.9 \%, \mathrm{TPN}+\mathrm{FOR}=18.8 \%$ ).

Nuclear HIF1 $\alpha$-IR was often found in enterocytes exhibiting a more intense staining at the villus tip and fading toward the crypts, sometimes co-stained with lamina propria cells (Fig. 3). Nuclear HIF1 $\alpha$ staining was clearly associated with the administration of formula $(p=0.01)$ and occurred more in the caudal region of enterally fed piglets $(p=0.02)$ (Fig. 4). NEC lesions were not associated with nuclear HIF1 $\alpha$ staining.

Villus growth. Mucosal growth was taken into consideration, since age and feeding could be expected to have an impact on intestinal villus volume, and thus microvascular density. Region and treatment interacted significantly in regards to villus volume. In the cranial region, only the TPN group showed significant villus growth ( $+94 \%$ compared with $\mathrm{NB}, p=0.02)$, in agreement with previous reports $(17,21)$. In the caudal region, villus volume of TPN + SOW piglets showed a remarkable increase compared with TPN piglets $(+128 \%, p<0.01)$ and TPN + FOR piglets $(+64 \%, p<0.05)$ (Fig. 5).

Volume density of $v W F$ (proportion of endothelial mass in the intestinal wall). The most dense endothelial distribution was observed in the mucosal layer $($ Vvmuc $=14.0 \pm 0.3 \%)$. The submucosal and muscle layer were less densely vascularized $($ Vvsubmuc $=9.2 \pm 0.3 \%$; Vvmusc $=6.2 \pm 0.3 \%)$. Of all vWF-IR in the intestinal wall, $81.0 \%$ was present in the mucosal layer, which can be attributed to the finding that the mucosal layer occupies $73.8 \pm 0.8 \%$ of the intestinal wall in these piglets (21).
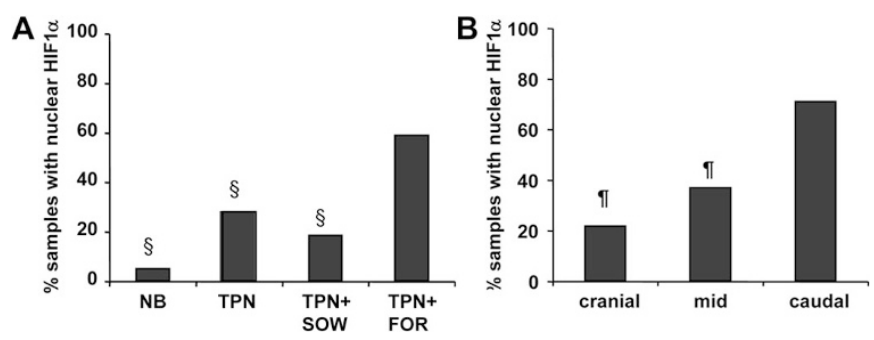

Figure 4. Occurrence of nuclear HIF $1 \alpha$. Percentage of intestinal samples expressing nuclear HIF1 $\alpha$ in enterocytes are shown according to different feedings $(A)$ and intestinal regions of enterally fed piglets $(B)$. Formula feeding was associated with more nuclear HIF $1 \alpha$ staining than nonenterally fed or colostrum piglets. There was more nuclear HIF1 $\alpha$ staining in the caudal region of enterally fed piglets compared with more cranial regions in these piglets. $\S p<0.05$ compared with TPN+FOR; I $p<0.05$ compared with caudal region. 


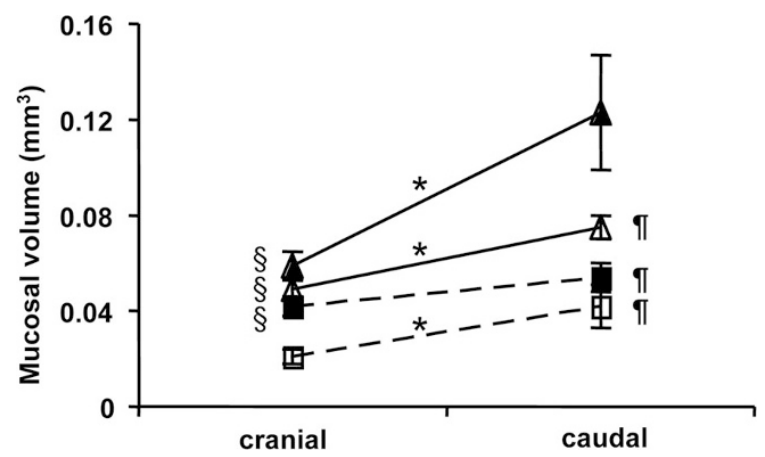

Figure 5. Absolute villus volume. Cranial and caudal villus volume is shown according to feeding type $(\square=\mathrm{NB}, \boldsymbol{\square}=\mathrm{TPN}, \boldsymbol{\Delta}=\mathrm{TPN}+\mathrm{SOW}, \Delta=$ TPN+FOR). Nonenterally fed pigs (NB, TPN) are shown as a dashed line, enterally fed pigs as a solid line. Values are means \pm SEM. $\S p<0.05$ compared with $\mathrm{NB} ; \mathbb{I} p<0.05$ compared with $\mathrm{TPN}+\mathrm{SOW}$ *significant cranio-caudal gradient $(p<0.05)$.

Irrespective of feeding regimen, mucosal vWF density decreased linearly from the cranial $(15.9 \pm 0.4 \%)$ to the caudal $(11.8 \pm 0.5 \%)$ small intestine (Fig. $6 A)(p<0.01)$. In contrast, villus width showed a prominent cranial-to-caudal increase (from $78.4 \pm 1.8 \mu \mathrm{m}$ to $102.2 \pm 3.5 \mu \mathrm{m}, p<0.01$ ). Thus, a negative correlation was found between villus width and mucosal vWF density $(p<0.01)$, indicating that wider villi do not contain more vessels than smaller villi. Furthermore, a significant positive correlation was found between Vvmuc and former measurements of surface density (surface of the tunica mucosa divided by the volume of the tunica mucosa) (21) on the same intestinal biopsies $(p<0.05)$. Alterations in vWF density were not detected in the submu- cosal or muscle layer. No relation was found between mucosal vWF density and NEC lesions or nuclear HIF1 $\alpha$-IR in the intestine.

Volume density of eNOS (proportion of eNOS stained endothelium in the intestinal wall). The most dense eNOS-IR was displayed in the mucosal $(\mathrm{Vvmuc}=6.1 \pm 0.3 \%)$ and the submucosal (Vvsubmuc $=6.8 \pm 0.2 \%$ ) layer, whereas a smaller ratio was seen in the muscle layer (Vvmusc $=3.5 \pm$ $0.1 \%)$. Similar to vWF-IR, the largest portion of all eNOS-IR in the intestinal wall was present in the mucosal layer (78.9\%).

eNOS Vvmuc interacted statistically significant between feeding and region. The cranial small intestine showed no feeding-related differences; whereas, the middle and caudal small intestine of enterally fed piglets (TPN+FOR and TPN+SOW) had a lower mucosal eNOS-density compared with the nonenterally fed piglets (NB and TPN) (Fig. 7, Fig. $6 B$ ). This caused clear linear cranial-to-caudal decreasing gradients in enterally fed piglets (TPN+FOR, $p<0.01$; TPN + SOW, $p<0.05)$. In none of the other layers of the intestinal wall, feeding- or region-dependent effects were found. In necrotic tissue, Vvmuc was decreased compared with in healthy tissue $(p<0.05)$ (Fig. 8), but it was not related with presence or absence of HIF1 $\alpha$-IR (Fig. 9).

Volume density of eNOS relative to $v W F$ (proportion of eNOS-stained endothelium). Only a minor subset of endothelial cells expressed eNOS-IR in the intestinal mucosa (45.0 \pm $1.9 \%$ ), whereas almost the complete endothelium in the submucosal layer $(81.9 \pm 4.1 \%)$ and a large portion in the muscle layer $(63.1 \pm 4.5 \%)$ expressed eNOS-IR. Statistics performed on data from the mucosal layer showed an interaction between
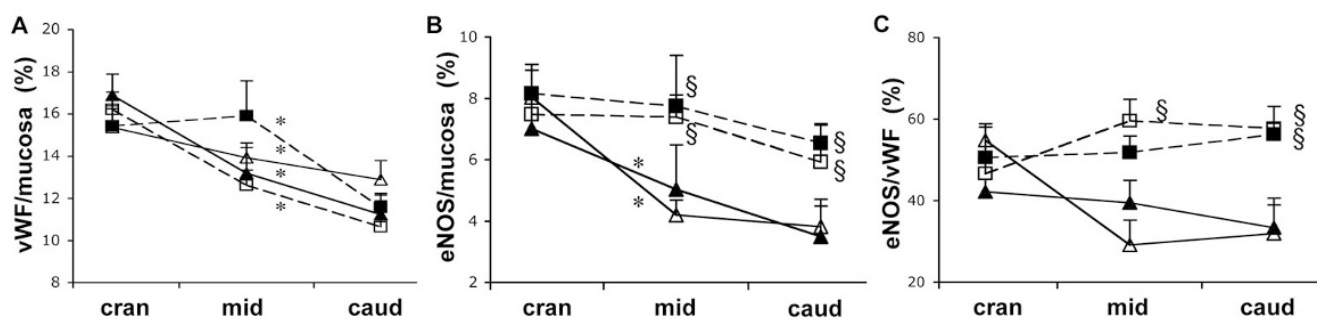

Figure 6. Mucosal volume densities Cranio-caudal decreasing gradients are shown, according to feeding type $(\square=\mathrm{NB}, \boldsymbol{\square}=\mathrm{TPN}, \boldsymbol{\Delta}=\mathrm{TPN}+\mathrm{SOW}, \Delta=$ TPN+FOR). Nonenterally fed pigs (NB, TPN) are shown as a dashed line, enterally fed pigs as a full line. Values are means + SEM. $\S p<0.05$ compared with TPN + FOR and TPN + SOW within region. *Significant cranio-caudal gradient $(p<0.05)$. (A) Mucosal density of vWF decreases cranio-caudally, irrespective of feeding regimen. (B) A clear cranio-caudal decreasing gradient of endothelial nitric oxide synthase (eNOS)-volume relative to the mucosal volume can only be observed after enteral feeding, in contrast to the nonenterally fed groups. (C) Graph showing the percentage of eNOS-IR within vWF-IR for various regions and diets. A significant increasing cranio-caudal gradient can only be observed in NB. Enterally fed piglets show lower eNOS/vWF density in the middle and caudal intestinal region compared with NB.
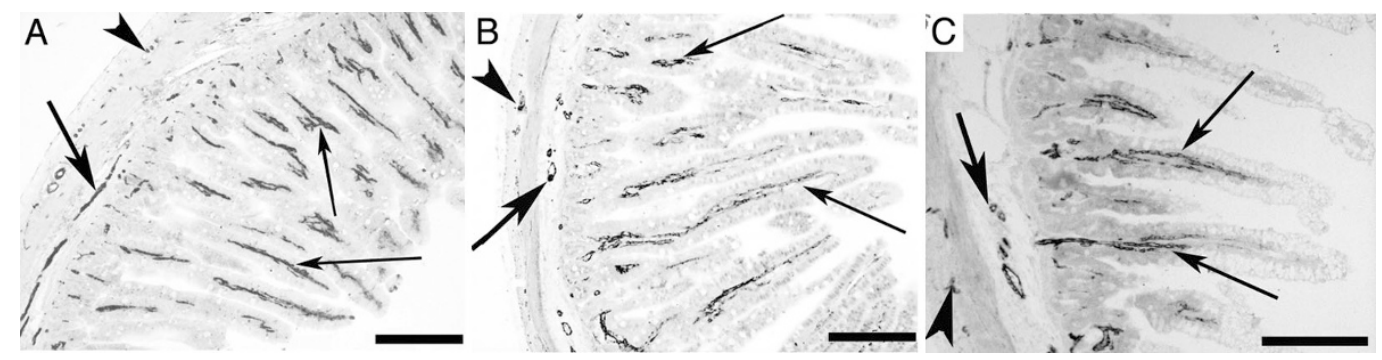

Figure 7. eNOS immunoreactivity according to different feeding regimens. Immunostaining for eNOS in the distal small intestinal region of piglets receiving $3 \mathrm{~d}$ of total parenteral nutrition (TPN) $(A)$, or $3 \mathrm{~d}$ of TPN followed by $2 \mathrm{~d}$ of either sow's colostrum $(B)$ or formula $(C)$. eNOS-IR is present in small vessels in the muscles (arrowheads), larger vessels in the submucosa (thick arrows) and capillaries in the villi (thin arrows). In formula-fed piglets, eNOS-IR in the villus tips is scarce, despite a clear expression at the basis of the villi $(C)$. Scale bar $=250 \mu \mathrm{m}$. 


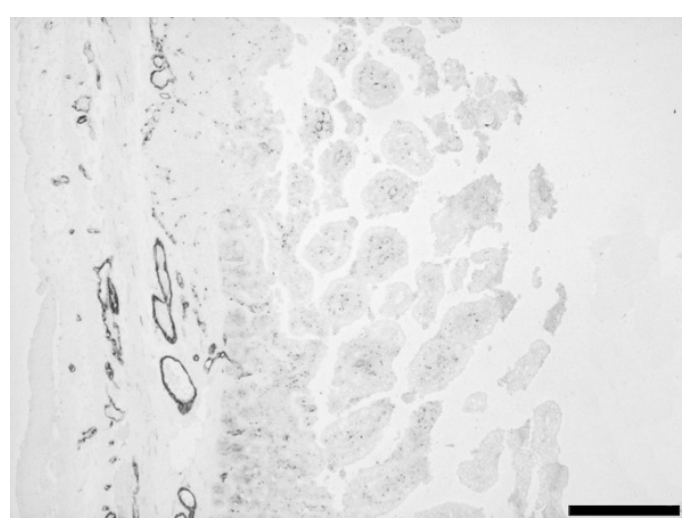

Figure 8. eNOS immunoreactivity at NEC-like lesions. eNOS in the small intestine of a formula-fed piglet with necrotic lesions appears low and scattered in the villi. Scale bar $=200 \mu \mathrm{m}$.
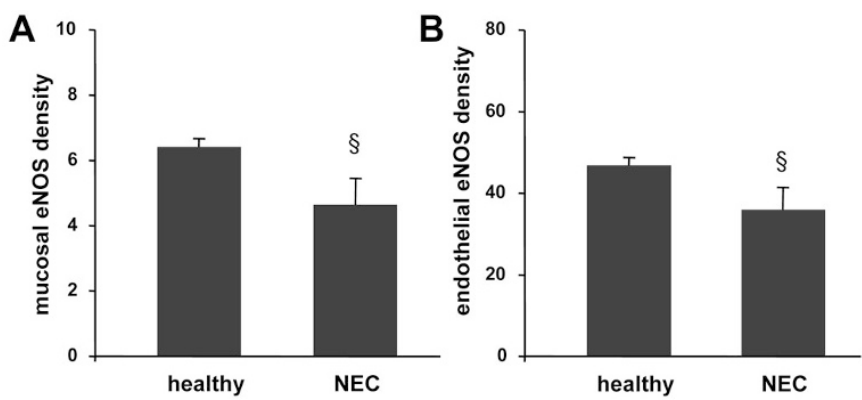

Figure 9. eNOS volume densities in NEC. Mucosal eNOS density is lower in intestinal samples showing NEC lesions $(A)$. This is due to a lower endothelial eNOS expression and not because of mucosal volume alterations, as endothelial eNOS density is also lower in necrotic tissue $(B) . \S p<0.05$ compared with nonnecrotic, healthy tissue.

intestinal regions and feeding regimen. No clear gradients along the small intestine were observed, except a tendency to decreasing cranio-caudal percentages in TPN + FOR piglets $(p=0.06)$. The divergence of the diet-dependent gradients along the small intestine lead to significant differences in the middle (TPN versus TPN+FOR) and caudal region only (TPN versus TPN+FOR and TPN+SOW) (Fig. 6C). Necrotic lesions in the intestine were associated with a significantly decreased ratio in mucosal eNOS/vWF, compared with healthy tissue (Fig. 9, $p<0.05$ ) but no relation was observed with the presence of nuclear HIF1 $\alpha$-IR.

\section{DISCUSSION}

Alterations in the intestinal endothelium (vWF) and endothelial vasodilatory capacity (eNOS). Following birth, the intestinal circulation has to cope with an increased mucosal oxygen demand at the moment of enteral feeding and suffice for nutrient absorption (5). In addition, it is challenged by the enormous growth rate of the intestinal mucosa during the first days of life. Surprisingly, the distribution and growth of the intestinal microvasculature in the neonatal intestine is scarcely documented. The present results document for the first time a clear cranial-to-caudal decrease in microvasculature density. The negative correlation between mucosal vWF density and villus width suggests a cranial-to-caudal tissue dilution effect that makes the absolute amount of capillaries per villus the same in the cranial and caudal small intestine while villi are wider in the caudal region. An explanation may be related to the fountain-like distribution of villus capillaries (20), where downstream capillaries are uniformly spread against the abluminal surface of enterocytes. Since small villi have an enlarged surface compared with broader villi containing a similar volume, it is likely that more downstream capillaries are present in a volume of smaller villi (cranial region) compared with broader villi (caudal region) to exhibit a similar spread along the surface. This is supported by the observed negative correlation between mucosal vWF density and surface density, although we cannot exclude the presence of wider capillaries in the caudal small intestine. A cranial-to-caudal decrease in intestinal blood flow has been reported in dogs (11), pigs $(12,13)$, and humans (14). Based on our data, we suggest that blood flow gradients along the small intestine are related to the cranial-to-caudal decreasing gradients in mucosal vWF and/or eNOS, an endothelial enzyme responsible for the production of the main vasodilator NO in neonatal and premature animals $(5,7,9)$. Furthermore, this blood flow gradient was prominent in the mucosal layer only (11), which is in agreement with the absence of an eNOS- or vWF gradient in the submucosal and muscle layer.

We investigated the intestinal characteristics over 2-3 d of TPN combined with 1-2 d of enteral feeding with colostrum or formula. Our design did not allow for a detailed analysis of both age and diet effects but the results on mucosal vWF density indicate that during the first days of premature life, the intestinal endothelium grows with a rate similar to the intestinal mucosa and this growth is not affected by type of diet (TPN, colostrum, or formula). In contrast, the expression of mucosal endothelial eNOS is diet- and region-dependent. In the cranial region, it remained unchanged during all feedings, although TPN piglets showed significant mucosal growth in this region. Therefore, an equal rate of increasing eNOS-IR to the mucosal growth occurs in TPN piglets. In the middle and caudal small intestinal mucosa however, eNOS density in TPN + FOR and TPN+SOW piglets decreased, compared with TPN piglets. Probably, the eNOS-IR lagged behind the remarkable growth spurt of the villi in the caudal intestinal region of TPN + SOW piglets, whereas VWF-IR showed a similar growth rate. ENOS-IR was also reduced in the middle and caudal region of TPN+FOR piglets, despite limited mucosal growth. It is therefore possible that formula-induced microbial changes or inflammatory responses are involved $(3,22)$, as these factors are known to decrease eNOS-protein expression $(23,24)$ and predominate in the caudal small intestine (25). Some compensation for this low eNOS density in enterally fed piglets may have occurred by an increased eNOS expression in terminal mesenteric arteries, as described previously in term piglets following enteral feeding (7).

Endothelial alterations in relation to NEC. Since the caudal intestinal region of enterally fed piglets is characterized by a low microvascular density and low relative eNOS expression, this region is prone to develop hypoxia as soon as it becomes further stressed. This may explain why lesions in the neonatal small intestine under stressed or hypoxic conditions occur primarily in the caudal small intestine of rats (26-28) 
and pigs $(13,29,30)$. Similarly, in the present piglet model and in human NEC, lesions occur preferentially in the caudal regions, especially when the intestine has to cope with an inferior feeding such as formula $(2,31)$. Formula may be more provocative to the intestine, than colostrum, because it lacks the natural immunomodulatory factors (32), resulting in inflammatory responses (22) and bacterial overgrowth (3). The existence of such stress in premature formula-fed piglets was indicated by the presence of nuclear HIF1 $\alpha$-IR, a stress-sensitive cellular marker. In addition, the caudal small intestine of enterally fed piglets was exposed to more nuclear HIF1 $\alpha$ inducing insults, probably related to low eNOS density - thus high hypoxia sensitivity-in this region. On the other hand, the lack of a direct relationship indicates that additional stress factors have been involved $(15,16)$. A direct association between NEC lesions and low eNOS expression was detected, similar to human NEC (33) where hypoxia is known to be a contributing factor (6). Therefore, we speculate that a low eNOS density increases the risk for NEC by increasing the hypoxia sensitivity of the intestine.

In conclusion, the mucosal endothelial density along the small intestine in premature piglets decreases cranio-caudally, regardless of feeding regimen (TPN or enteral), whereas for mucosal eNOS expression, such a gradient is present only when enteral feeding is initiated. We hypothesize a delayed growth response of eNOS-IR in the fast growing mucosa caudally in the intestine of TPN + SOW and a down-regulating effect of stress-factors associated with formula, as shown by nuclear HIF1 $\alpha$, on eNOS expression in TPN+FOR. The increased NEC sensitivity in enterally fed preterm pigs may thus in part be explained by increased hypoxia sensitivity of caudal intestinal regions resulting from a decreased mucosal eNOS density.

Acknowledgments. The authors thank Katty Huybrechts for her technical assistance and support.

\section{REFERENCES}

1. Berseth CL, Bisquera JA, Paje VU 2003 Prolonging small feeding volumes early in life decreases the incidence of necrotizing enterocolitis in very low birth weight infants. Pediatrics 111:529-534

2. Lucas A, Cole TJ 1990 Breast milk and neonatal necrotising enterocolitis. Lancet 336:1519-1523

3. Sangild PT, Siggers RH, Schmidt M, Elnif J, Bjornvad CR, Thymann T, Grondahl ML, Hansen AK, Jensen SK, Boye M, Moelbak L, Buddington RK, Westrom BR, Holst JJ, Burrin DG 2006 Diet- and colonization-dependent intestinal dysfunction predisposes to necrotizing enterocolitis in preterm pigs. Gastroenterology 130:17761792

4. Greer FR 2001 Feeding the premature infant in the 20th century. J Nutr 131:426S$430 \mathrm{~S}$

5. Reber KM, Nankervis CA, Nowicki PT 2002 Newborn intestinal circulation. Physiology and pathophysiology. Clin Perinatol 29:23-39

6. Nowicki PT 2005 Ischemia and necrotizing enterocolitis: where, when, and how Semin Pediatr Surg 14:152-158

7. Reber KM, Su BY, Clark KR, Pohlman DL, Miller CE, Nowicki PT 2002 Developmental expression of eNOS in postnatal swine mesenteric artery. Am J Physiol Gastrointest Liver Physiol 283:G1328-G1335
8. Fan WQ, Smolich JJ, Wild J, Yu VY, Walker AM 1996 Nitric oxide modulates regional blood flow differences in the fetal gastrointestinal tract. Am J Physiol 271:G598-G604

9. Nankervis CA, Nowicki PT 1995 Role of nitric oxide in regulation of vascular resistance in postnatal intestine. Am J Physiol 268:G949-G958

10. Forstermann U, Boissel JP, Kleinert H 1998 Expressional control of the 'constitutive' isoforms of nitric oxide synthase (NOS I and NOS III). FASEB J 12:773-790

11. Dinda PK, Beck IT 1986 Arteriolar, capillary, and shunt flow gradients along the intestine in relation to the weight distribution of the mucosa, submucosa, and muscularis-a microsphere study. Can J Physiol Pharmacol 64:1127-1136

12. Nowicki PT, Stonestreet BS, Hansen NB, Yao AC, Oh W 1983 Gastrointestinal blood flow and oxygen consumption in awake newborn piglets: effect of feeding. Am J Physiol 245:G697-G702

13. Sibbons PD, Spitz L, van Velzen D 1992 Collateral blood flow in the distal ileum of neonatal piglets: a clue to the pathogenesis of necrotizing enterocolitis. Pediatr Pathol 12:15-27

14. Geber WF 1960 Quantitative measurement of blood flow in various areas of small and large intestine. Am J Physiol 198:985-986

15. Koury J, Deitch EA, Homma H, Abungu B, Gangurde P, Condon MR, Lu Q, Xu DZ, Feinman R 2004 Persistent HIF-1alpha activation in gut ischemia/reperfusion injury: potential role of bacteria and lipopolysaccharide. Shock 22:270-277

16. Scharte M, Han X, Bertges DJ, Fink MP, Delude RL 2003 Cytokines induce HIF-1 DNA binding and the expression of HIF-1-dependent genes in cultured rat enterocytes. Am J Physiol Gastrointest Liver Physiol 284:G373-G384

17. Sangild PT, Petersen YM, Schmidt M, Elnif J, Petersen TK, Buddington RK, Greisen G, Michaelsen KF, Burrin DG 2002 Preterm birth affects the intestinal response to parenteral and enteral nutrition in newborn pigs. J Nutr 132:3786-3794

18. Skrzypek T, Valverde Piedra JL, Skrzypek H, Wolinski J, Kazimierczak W, Szymanczyk S, Pawlowska M, Zabielski R 2005 Light and scanning electron microscopy evaluation of the postnatal small intestinal mucosa development in pigs. J Physiol Pharmacol 56:71-87

19. Gundersen HJ, Jensen EB 1987 The efficiency of systematic sampling in stereology and its prediction. J Microsc 147:229-263

20. Bellamy JE, Latshaw WK, Nielsen NO 1973 The vascular architecture of the porcine small intestine. Can J Comp Med 37:56-62

21. Oste M, Van Ginneken CJ, Van Haver ER, Bjornvad CR, Thymann T, Sangild PT 2005 The intestinal trophic response to enteral food is reduced in parenterally fed preterm pigs and is associated with more nitrergic neurons. J Nutr 135:2657-2663

22. Minekawa R, Takeda T, Sakata M, Hayashi M, Isobe A, Yamamoto T, Tasaka K, Murata Y 2004 Human breast milk suppresses the transcriptional regulation of IL-1beta-induced NF-kappaB signaling in human intestinal cells. Am J Physiol Cell Physiol 287:C1404-C1411

23. Chen K, Inoue M, Wasa M, Fukuzawa M, Kamata S, Okada A 1997 Expression of endothelial constitutive nitric oxide synthase mRNA in gastrointestinal mucosa and its downregulation by endotoxin. Life Sci 61:1323-1329

24. Cardaropoli S, Silvagno F, Morra E, Pescarmona GP, Todros T 2003 Infectious and inflammatory stimuli decrease endothelial nitric oxide synthase activity in vitro. J Hypertens 21:2103-2110

25. Shirkey TW, Siggers RH, Goldade BG, Marshall JK, Drew MD, Laarveld B, Van Kessel AG 2006 Effects of commensal bacteria on intestinal morphology and expression of proinflammatory cytokines in the gnotobiotic pig. Exp Biol Med (Maywood) 231:1333-1345

26. Nadler EP, Dickinson E, Knisely A, Zhang XR, Boyle P, Beer-Stolz D, Watkins SC, Ford HR 2000 Expression of inducible nitric oxide synthase and interleukin-12 in experimental necrotizing enterocolitis. J Surg Res 92:71-77

27. Dvorak B, Halpern MD, Holubec H, Williams CS, McWilliam DL, Dominguez JA, Stepankova R, Payne CM, McCuskey RS 2002 Epidermal growth factor reduces the development of necrotizing enterocolitis in a neonatal rat model. Am J Physiol Gastrointest Liver Physiol 282:G156-G164

28. Dimmitt RA, Glew R, Colby C, Brindle M, Skarsgard E, Moss RL 2003 Serum cytosolic beta-glucosidase activity in a rat model of necrotizing enterocolitis. Pediatr Res 54:462-465

29. Ewer AK 2002 Role of platelet-activating factor in the pathophysiology of necrotizing enterocolitis. Acta Paediatr Suppl 91:2-5

30. Thornbury JC, Sibbons PD, van Velzen D, Trickey R, Spitz L 1993 Histological investigations into the relationship between low birth weight and spontaneous bowel damage in the neonatal piglet. Pediatr Pathol 13:59-69

31. Sangild PT 2006 Gut responses to enteral nutrition in preterm infants and animals. Exp Biol Med (Maywood) 231:1695-1711

32. Newburg DS, Walker WA 2007 Protection of the neonate by the innate immune system of developing gut and of human milk. Pediatr Res 61:2-8

33. Nowicki PT, Reber KM, Giannone PJ, Nankervis CA, Hammond S, Besner GE, Caniano DA 2006 Intestinal O2 consumption in necrotizing enterocolitis: role of nitric oxide. Pediatr Res 59:500-505 